\title{
Determinantes da participação e dos rendimentos dos jovens no mercado de trabalho: o caso da Região Metropolitana de Recife
}

\author{
Determinants of young people's participation and income \\ in the labor market: the case of the Metropolitan Area of Recife \\ Carolina Rodrigues Corrêa \\ João Eustáquio de Lima
}

\begin{abstract}
Resumo
0 presente artigo buscou analisar os determinantes da inserção dos jovens da Região Metropolitana de Recife (RMR) no mercado de trabalho, bem como os determinantes dos rendimentos daqueles que trabalham. Para tanto, foi estimada uma equação utilizando o procedimento de Heckman com dados da PNAD de 2012. Os resultados mostram que ser do sexo masculino e chefe da família, maior idade e a escolaridade afetam positivamente a decisão do jovem de entrar no mercado de trabalho. Para os rendimentos, ser do sexo masculino, branco e maior escolaridade faz com que esses sejam maiores. Assim sendo, investir na educação e nas condições de acesso à mesma é um passo indispensável rumo à redução das disparidades sociais da Região Metropolitana de Recife.
\end{abstract}

Palavras-chave: jovens; mercado de trabalho; rendimentos; educação; desenvolvimento.

\begin{abstract}
This article analyzes the determinants of the entrance of young people from the Metropolitan Area of Recife in the labor market, as well as the determinants of the income received by those who work. An equation was estimated using the procedure proposed by Heckman for sample selectivity, using data from PNAD (2012). The results show that being male and the head of the family, being a major, and having a higher level of schooling positively affect the young adult's decision to enter the labor market. As for the determination of income, being male, white and having a higher level of schooling make the income be higher. Therefore, investing in young people's education and in their access to it is an indispensable step towards reducing social disparities in the Metropolitan Area of Recife.
\end{abstract}

Keywords: young people; labor market; income; education; development. 


\section{Introdução}

A participação dos jovens no mercado de trabalho é bastante discutida por pesquisadores e formuladores de políticas públicas. A importância desse tema reside nas implicações de começar a trabalhar na vida dos jovens e suas famílias. Como em muitos casos trabalho e estudo são atividades de difícil conciliação, muitos jovens trabalhadores optam por abandonar os estudos, o que no longo prazo pode significar redução drástica das oportunidades de desenvolvimento econômico e social desses.

A juventude do Brasil é trabalhadora, e suas taxas de participação no mercado de trabalho são relativamente altas. Também é elevada a parcela daqueles que buscam conciliar os estudos e o trabalho ou que transitam de uma situação a outra (ONU BR, 2014). De acordo com Mesquita (2011), é comum que os jovens brasileiros ingressem cedo no mercado de trabalho. Tal fato, muitas vezes, pode acarretar em abandono dos estudos.

Silva e Oliveira (2007) afirmam que uma parcela dos jovens é poupada da inserção no mercado de trabalho por estar na escola. Outra parcela já está inserida no mercado de trabaIho e tem que conciliar educação e trabalho. Por fim, existem ainda muitos jovens que abandonam os estudos para trabalhar, o que compromete o processo de formação e também de capacitação profissional.

Saraví (2009) argumenta que a renda familiar e as condições do lar de origem dos jovens influenciam no seu desempenho escolar e no acesso ao ensino médio. Para os jovens dos estratos sociais mais elevados, a conclusão do ensino médio é de grande importância e é estimulada pela família. Porém, para os jovens de menor renda, a escola, particularmente o ensino médio e superior, se torna menos importante.

Assim, o que se observa é que, no caso de famílias com estrato de renda mais baixo, a inserção precoce do jovem no trabalho, ao acarretar abandono dos estudos, pode significar manutenção do nível de pobreza da família.

Segundo a OIT (2009), muitos jovens brasileiros ingressam no mercado de trabalho por necessidade econômica de suas famílias. Por outro lado, esse estudo também indica que há jovens que ingressam no mercado de trabalho por desejarem autonomia, independência financeira, além de crescimento profissional e pessoal.

Porém, ao decidir entrar no mercado de trabalho os jovens encontram muitos empeciIhos, tanto para conseguir o primeiro emprego quanto para mudar de ocupação. De acordo com Mesquita (2011), em muitos casos, esses acabam por ocupar os piores postos de trabaIho. Entre as exigências do mercado de trabaIho para contratação está a formação escolar e profissional, bem como a experiência adquirida em outros postos de trabalho. No caso dos jovens, essas duas exigências são difíceis de atender, visto que estão passando por um processo de formação escolar e profissional, e têm pouca ou nenhuma experiência profissional.

Logo, é de se esperar que os jovens, ao optarem por trabalhar, se deparem com salários relativamente baixos e colocações em postos de trabalho inferiores. Sendo assim, torna-se importante compreender os motivos que levam os jovens a optar por trabalhar, além de verificar os fatores que afetam o nível de rendimento dos jovens que optam por fazê-lo. Com 
isso, é possível determinar em quais frentes atuar para possibilitar aos mesmos melhores oportunidades de crescimento.

\section{Trabalho juvenil, educação e desenvolvimento socioeconômico}

De acordo com Barros et al. (2012), a sustentabilidade do desenvolvimento socioeconômico está diretamente associada à agilidade e à continuidade do processo de expansão da educação. Maior educação aumenta a produtividade do trabalho, contribuindo para o crescimento econômico, elevação de salários e redução da pobreza. Assim, a educação serve de instrumento de mobilidade social.

Desenvolver a região Nordeste e reduzir as desigualdades econômicas e sociais, bem como eliminar a pobreza sempre foram pontos presentes nas pautas dos formuladores de políticas públicas. Assim sendo, para obter êxito nas políticas de desenvolvimento do Nordeste (e outras regiões), proporcionar aos jovens oportunidades de se educarem mais se torna imprescindível. Porém, muitas vezes a necessidade de trabalhar faz com que esses não possam de dedicar aos estudos.

Devido à importância da educação para o crescimento pessoal e desenvolvimento regional, e da existência de um trade off entre trabalhar e estudar para muitos jovens, torna-se importante verificar quais os fatores mais importantes na decisão dos jovens de entrar no mercado de trabalho. Optou-se por analisar a região Nordeste, devido a suas grandes disparidades econômicas e elevados índices de pobreza. Mais especificamente, foi objeto desse estudo a Região Metropolitana de Recife (RMR), por sua grande expressão na região Nordeste e no cenário nacional.

A RMR foi institucionalizada em 1973 e, de acordo com dados do Censo de 2010, é o maior aglomerado urbano do Nordeste e a $5^{\mathrm{a}}$ região mais populosa do Brasil. Tem a maior parte do PIB estadual $(65,1 \%)$, além de $42 \%$ da população de Pernambuco. Desse total, $97,26 \%$ dos habitantes são residentes da zona urbana e $2,74 \%$ moram na zona rural (Bitoun et al., 2012).

De acordo com dados da Pesquisa Nacional por Amostra de Domicílios (PNAD) de 2012, realizada pelo Instituto Brasileiro de Geografia e Estatística (IBGE), cerca de $45 \%$ dos jovens entrevistados da RMR, entre 16 e 25 anos, trabalharam na semana em que a pesquisa foi realizada, ${ }^{1}$ como é possível observar na Figura 1.

A Tabela 1 mostra a posição na ocupação dos jovens da região. É possível observar que a grande maioria dos jovens que estavam trabalhando $(83,59 \%)$ era de funcionários (empregados).

Assim sendo, é possível concluir que uma grande parte dos jovens da Região Metropolitana de Recife optou por trabalhar, o que torna importante estudar a questão para a referida região. Portanto, o presente artigo busca verificar quais são os determinantes da participação dos jovens no mercado de trabaIho na RMR e identificar quais são os fatores que determinam o nível salarial recebido por esses jovens trabalhadores. 
Figura 1 - Percentual de jovens trabalhando na Região Metropolitana de Recife em 2012

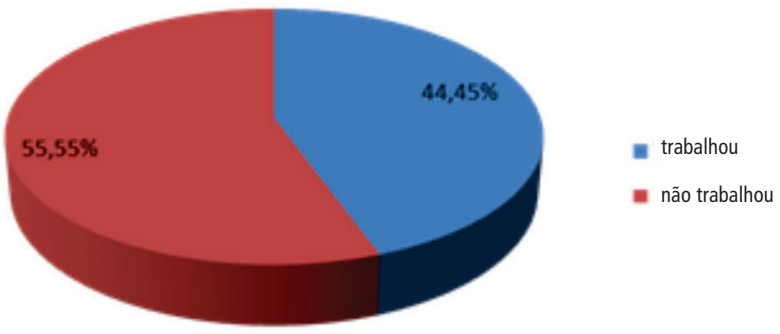

Fonte: Elaboração própria com dados da PNAD.

Tabela 1 - Posição na ocupação dos jovens da RMR que estavam trabalhando em 2012

\begin{tabular}{l|c}
\hline \multicolumn{1}{c|}{ Posição na ocupação } & $\%$ \\
\hline Empregado & 83,59 \\
Trabalhador doméstico & 4,1 \\
Conta própria & 10,55 \\
Empregador & 0,49 \\
Trabalhador não remunerado membro da unidade domiciliar & 0,88 \\
Outro trabalhador não remunerado & 0,2 \\
Trabalhador na construção para o próprio uso & 0,2 \\
\hline
\end{tabular}

Fonte: Elaboração própria com dados da PNAD. 


\section{Referencial teórico}

De acordo com Scorzafave e Menezes-Filho (2001), a decisão de participação no mercado de trabalho está ligada à oferta de trabalho. Com base na teoria neoclássica da oferta de trabalho, o indivíduo deseja maximizar sua utilidade com o consumo de bens e horas de lazer, sujeito à renda não oriunda do trabalho, preço dos bens, preço do lazer e tempo disponível.

Ainda segundo os autores, dado esse problema de maximização, conclui-se que a Taxa Marginal de Substituição (TMS) entre consumo de bens e horas de lazer pode ser entendida como o salário de reserva do indivíduo, ou seja, quanto esse requer de remuneração adicional para perder uma hora de lazer. Assim, pode-se definir que o indivíduo participará da força de trabalho sempre que o salário de mercado relativo (preço do lazer/preço dos bens) exceder seu salário de reserva (TMS).

No caso dos jovens, outros fatores são considerados ao tomar a decisão de trabalhar. Souza et al. (2011) assinalam a renda familiar como um determinante. Espera-se que com rendas mais altas, a pressão para o jovem iniciar uma atividade profissional seja menor. Altonji et al. (2012) apontam a escolaridade dos pais como fator importante. Pais com maior escolaridade tendem a valorizar mais os estudos, incentivando o jovem a dedicar mais tempo a essa atividade. Outra variável, de acordo com Basu e Van (1998), seria o tamanho da família. Famílias mais numerosas tendem a necessitar do trabalho do jovem para complementar a renda.

Outros fatores como idade, sexo, estado civil, se há ou não crianças (que não geram renda) na família e escolaridade são aspectos importantes para o ingresso do jovem no mercado de trabalho. Idade, ser do sexo masculino, ser casado(a) e ter crianças dependentes na família afetaria positivamente a probabilidade de estar trabalhando, visto que a necessidade e as pressões para contribuir com a renda familiar aumentam. A escolaridade também afetaria positivamente. Farber e Gibbons (1996) afirmam que, quanto maior a escolaridade, maior seria a probabilidade de o jovem trabalhar devido a probabilidade de empregos com salários mais altos.

Com relação à determinação dos rendimentos do trabalhador, baseia-se na teoria do capital humano. Mincer (1974) estudou os impactos do capital humano sobre os rendimentos, buscando a correlação entre o investimento na formação do trabalhador e a distribuição da renda. Seu trabalho visou medir o impacto do incremento de um ano de estudo sobre o salário. 0 autor concluiu que a produtividade e, por conseguinte, as diferenças de rendimentos entre os indivíduos estariam positivamente relacionadas ao volume do investimento em capital humano de cada um.

Lima (1980) também afirma que o rendimento de um indivíduo está ligado ao capital humano, que seria fruto do investimento, especialmente em educação, dos anos de experiência e das diferentes habilidades cognitivas e características pessoais dos indivíduos. Assim, haveria diferenças na qualidade da mão de obra de cada um. No caso dos jovens, considerar a experiência não faz sentido, visto que os mesmos têm pouca ou nenhuma.

Becker (1971) apronta para a discriminação no mercado de trabalho, no que tange a características que não afetam a produtividade do trabalhador. A discriminação gera salários 
desiguais e tratamento diferenciado relacionado a fatores não produtivos, como sexo e raça. Coelho e Corseuil (2002) apontam como fator importante também a região na qual os trabalhadores estão inseridos, uma vez que economias com maior nível de desenvolvimento tendem a possibilitar uma maior remuneração para o trabalho.

Diversos estudos buscaram analisar os determinantes de rendimentos dos trabalhadores de diversas características deferentes, como os de Langoni (1973), Suliano e Siqueira (2012) e Leone e Baltar (2013).

Langoni (1973) estimou seu modelo com equações log-lineares utilizando como variáveis explicativas a educação, idade, sexo, atividade e região de residência. 0 autor considerava que a educação é fortemente correlacionada com a renda.

Suliano e Siqueira (2012) estimaram uma equação de salários para as regiões Sudeste e Nordeste do Brasil, para os anos de 2001 a 2006, e as evidências mostraram que o retorno da escolaridade se mantém em patamares elevados.

Leone e Baltar (2013) analisam a diversidade de rendimentos do trabalho, examinando a contribuição das diferenças de escolaridade, sexo e posição na ocupação. Os autores concluíram que as diferenças de rendimento por sexo, embora continuem bastante grandes, diminuíram.

\section{Metodologia}

Para analisar a decisão dos jovens da região metropolitana de Recife no mercado de trabalho, bem como a determinação dos rendimentos desses jovens trabalhadores, foi utilizado o Modelo de Seleção Amostral.

De acordo com Verbeek (2000), o modelo busca estimar duas equações, uma para definir a participação do indivíduo no evento e a outra para determinar o nível de uma variável relacionada à decisão.

No presente artigo, a primeira equação define se o jovem participa ou não do mercado de trabalho, enquanto a segunda determina 0 nível de rendimentos dos jovens.

0 procedimento, em dois estágios, foi proposto por Heckman. ${ }^{2}$ Baseando-se em Schlindwein e Kassouf (2006), no primeiro estágio estima-se um modelo Probit cuja variável dependente é 1 se o jovem estava trabalhando no período de referência e zero caso contrário.

$$
\begin{aligned}
& T=\alpha_{0}+\alpha_{1} \text { esc }+\alpha_{2} \text { idade }+\alpha_{3} \text { branco } \\
& +\alpha_{4} \text { sexo }+\alpha_{5} \text { casado }+\alpha_{6} \text { rendafam } \\
& +\alpha_{7} \text { tamanhofam }+\alpha_{8} \text { menores }+ \\
& \alpha_{9} \text { escpais }+\alpha_{10} \text { trenda }+\alpha_{11} \text { referencia }+ \\
& \alpha_{12} \text { trabalhochefe }+v
\end{aligned}
$$

em que $T=1$, se o jovem trabalhou e 0 , caso contrário (somente trabalho remunerado); esc é a escolaridade do jovem em anos de estudo; idade é a idade do jovem em anos; branco é uma dummy igual a 1 caso o jovem seja branco, 0 caso contrário; sexo é uma dummy igual a 1 caso sexo masculino, 0 caso contrário; casado é uma dummy igual a 1 caso o jovem seja casado, 0 caso contrário; rendafam é a renda da família em reais sem a renda do jovem; tamanhofam é o número de pessoas da família; menores é o número de pessoas com menos de 14 anos pertencentes à unidade familiar, escpais é a escolaridade dos pais em anos; trenda é uma 
dummy que indica se o jovem tem algum tipo de renda que não seja proveniente de trabalho, como juros, pensões, previdência, doação ou programas de transferência de renda, e é igual a 1 caso receba, 0 caso contrário; referência é uma dummy igual a 1 se o jovem é a pessoa de referência da família, 0 caso contrário; trabalhochefe é uma dummy igual a 1 se o chefe de família trabalha e 0 caso contrário; $v$ é o termo de erro.

No segundo estágio, estima-se a equações de rendimentos que pode ser expressa da seguinte forma:

$\ln W=\beta_{0}+\beta_{1}$ esc $+\beta_{2}$ sexo $+\beta_{3}$ idade + $\beta_{4}$ branco $+\beta_{5}$ setorprim $+\beta_{6}$ setorsec + $\beta_{7}$ setorter $+\beta_{8} R I M+\mu$

em que InW é o logaritmo do rendimento do indivíduo; esc, sexo, idade e branco são as mesmas do primeiro estágio; setorprim é uma dummy igual a 1 caso o jovem trabalhe no setor primário, 0 caso contrário; setorsec é uma dummy igual a 1 caso o jovem trabalhe no setor secundário, 0 caso contrário; setorter é uma dummy igual a 1 caso o jovem trabalhe no setor terciário, 0 caso contrário; $R / M$ é razão inversa de Mills, gerada pela regressão probit no primeiro estágio; $\mu$ é o termo de erro.

A razão inversa de Mills é uma variável gerada pelo próprio modelo com o intuito de corrigir o viés de seleção amostral.

Posteriormente são calculados os efeitos marginais para verificar o efeito de cada variável explicativa sobre cada variável dependente.

Os dados utilizados nesse artigo são oriundos da PNAD 2012, realizada pelo IBGE. Considerou-se como jovens os indivíduos entre
16 e 25 anos de idade. Tal corte etário foi escolhido pois: 1) o corte inferior, 16 anos, é a idade legal em que o jovem pode trabalhar no Brasil; 2) o corte superior é uma idade de transição para o jovem. Grande parte dos que optaram por fazer um curso superior já concluíram ou o farão em breve, assim, aqueles que não trabalham vão procurar um emprego e os que já trabalharam podem buscar um emprego meIhor; e 3) essa faixa etária é de grande vulnerabilidade no que tange o desemprego, devido à pouca experiência.

Como a PNAD é delineada como uma amostragem complexa, a estimação do modelo de seleção amostral foi realizada por meio do método de Pseudo Máxima Verossimilhança, que tende a gerar resultados mais consistentes. 0 software utilizado para a estimação foi o Stata 11.

\section{Resultados e discussão}

A Tabela 2 contém os resultados para a equação de participação no mercado de trabalho.

As variáveis escolaridade, idade, sexo e referência foram significativas e com sinal positivo, conforme esperado. Um ano a mais de escolaridade e idade aumentam, respectivamente, em 2,3 pontos percentuais (pp) e 6,55pp a probabilidade de o jovem trabaIhar. Maior escolaridade eleva as chances de conseguir uma melhor colocação, já ser mais velho faz com que uma maior responsabilidade recaia sobre o jovem. Ser do sexo masculino e ser a pessoa de referência da família aumentam, respectivamente, 23,03pp e 16,99pp. Ambos afetam também a pressão 
Tabela 2 - Probabilidade do jovem da RMR estar trabalhando em 2012

\begin{tabular}{l|c|c|c}
\hline \multicolumn{1}{c|}{ Variável } & Coeficiente & Erro padrão & Efeito Marginal Médio \\
\hline Escolaridade & $0,0601^{* * *}$ & 0,0123 & $0,0230^{* * *}$ \\
Idade & $0,1704^{* * *}$ & 0,0122 & $0,0655^{* * *}$ \\
Branco & $0,0843 \mathrm{~ns}$ & 0,0740 & $0,0231 \mathrm{~ns}$ \\
Sexo & $0,6089^{* * *}$ & 0,0683 & $0,2303^{* * *}$ \\
Casado & $0,2607 \mathrm{~ns}$ & 0,1839 & $0,1026 \mathrm{~ns}$ \\
Referência & $0,4308^{* * *}$ & 0,1467 & $0,1699^{* * *}$ \\
Trenda & $-0,3262^{*}$ & 0,1968 & $-0,1185^{*}$ \\
Rendafam & $-0,0364^{* *}$ & 0,0144 & $-0,0140^{* *}$ \\
Menores & $-0,0912 \mathrm{~ns}$ & 0,0794 & $-0,0348 \mathrm{~ns}$ \\
Tamanhofam & $0,0279 \mathrm{~ns}$ & 0,0243 & $0,0107 \mathrm{~ns}$ \\
Escpais & $-0,0271^{* * *}$ & 0,0081 & $-0,0104^{* * *}$ \\
Trabalhochefe & $0,0903 \mathrm{~ns}$ & 0,0697 & $0,0346 \mathrm{~ns}$ \\
Constante & $-4,5168^{* * *}$ & 0,2766 & - \\
\hline Estatística F & $18,40^{* * *}$ & & \\
\hline Y=1 & 0,3921 & & \\
\hline
\end{tabular}

*** significativo no nível de 1\%; ** significativo a 5\%; * significativo a $10 \%$; ns não significativo.

Fonte: Resultados da pesquisa.

para contribuir com a renda familiar. Se for a pessoa de referência, a necessidade de trabaIhar é ainda maior.

Analogamente, as variáveis trenda, rendafam e escpais também foram significativas e com sinal negativo, como se previa. 0 jovem receber transferência de renda reduz em $11,85 \mathrm{pp}$ a probabilidade de o mesmo trabaIhar, visto que a necessidade de auferir renda de outra fonte diminui. 0 aumento de uma unidade na renda da família gera uma queda de 1,40pp nessa probabilidade, assim como a escolaridade dos pais a reduz em 1,04pp por ano de estudo. Famílias com rendas mais altas podem dar suporte financeiro aos estudos do jovem, bem como pais com maior escolaridade tendem dar maior importância aos estudos.

A regressão é estatisticamente significativa, conforme o teste F. A probabilidade de o jovem estar trabalhando (remunerado) é, em média, 39,21\%.

A Tabela 3 apresenta os resultados do modelo de determinação dos rendimentos dos jovens. 
Tabela 3 - Determinantes dos rendimentos dos jovens da RMR em 2012

\begin{tabular}{l|c|c|c}
\hline \multicolumn{1}{c|}{ Variável } & Coeficiente & Erro padrão & Efeito Marginal Médio \\
\hline Idade & $0,1086^{* * *}$ & 0,0141 & $0,0510^{* * *}$ \\
Escolaridade & $0,0647^{* * *}$ & 0,0089 & $0,0445^{* * *}$ \\
Branco & $0,1038^{* *}$ & 0,0479 & $0,0755^{* *}$ \\
Sexo & $0,3146^{* * *}$ & 0,0516 & $0,1093^{* * *}$ \\
SetorPrim & $-0,4669^{* *}$ & 0,1893 & $-0,4669^{* * *}$ \\
SetorSec & $-0,0318 n s$ & 0,0526 & $-0,0318 n s$ \\
SetorTer & $-0,1227^{* * *}$ & 0,0402 & $-0,1227^{* * *}$ \\
Constante & $-2,0819^{* * *}$ & 0,4139 & - \\
\hline Estatística F & $18,40^{* * *}$ & & \\
\hline$\lambda$ & 0,4876 & 0,0769 & \\
\hline$\rho$ & 0,7972 & 0,0635 & \\
\hline
\end{tabular}

*** significativo no nível de 1\%; ** significativo a 5\%; * significativo a 10\%; ns não significativo. Fonte: Resultados da pesquisa.

As variáveis idade, escolaridade, branco e sexo foram positivas, conforme se esperava, e estatisticamente significativas. Um ano a mais de idade e escolaridade aumentam, respectivamente, $5,10 p p$ e 4,45pp o rendimento médio do jovem, refletindo a elevação do capital humano. Ser branco aumenta em 5,55pp, assim como ser do sexo masculino aumenta em 10,93pp, comprovando que ainda ocorre diferenciação de gênero e raça nos rendimentos dos jovens da RMR.

Setorprim e setorter também foram significativas. 0 sinal também foi o esperado, visto que os setores primário e terciário tendem a oferecer uma média salarial menor que a do setor secundário. Adicionalmente, a média salarial do setor primário tende a ser menor que a o terciário, como confirma a magnitude dos efeitos: trabalhar no setor primário reduz, em média, 46,69pp o rendimento do jovem, enquanto no setor terciário essa redução é de 12,27pp.

Hoffmann (2001) mostra que os salários na agricultura tendem a ser menores do que nos setores secundário e terciário da economia brasileira. De acordo com Medeiros (2011), o setor terciário setor sempre foi caracterizado por ter trabalhadores pouco produtivos e pagar salários baixos, comparando ao setor secundário. Isso não é necessariamente verdade para todos os subsetores, porém é possível verificar em vários deles. 
$\lambda$ e $\rho$ foram estatisticamente significativos, indicando que realmente há problema de viés de seleção amostral $(\lambda)$ e correlação relevante entre os erros das duas equações $(\rho)$, justificando o procedimento realizado.

Por fim, e equação possui significância global, visto que a estatística F foi significativa.

\section{Conclusões}

A participação dos jovens no mercado de trabalho é um assunto amplamente discutido em virtude de suas consequências para o futuro dos mesmos e para o desenvolvimento regional.

Apesar de, em muitos casos, a participação no mercado de trabalho se mostrar um empecilho aos estudos, foi possível observar que uma grande parcela dos jovens brasileiros e da RMR opta por trabalhar. Tal fato, se acarretar em abandono da educação, pode constituir um obstáculo ao crescimento do jovem e ao desenvolvimento da região.

0 modelo mostrou que, para os jovens da RMR, os fatores que influenciam positivamente na probabilidade de o jovem trabalhar são a idade, ser do sexo masculino e ser a pessoa de referência da família. Tais fatores trazem uma necessidade de maior responsabilidade do jovem, visto que esse se torna o provedor da família. Outro fator que afeta positivamente é a escolaridade. Isso ocorre porque uma maior escolaridade aumenta a empregabilidade desse jovem, por ser mais qualificado.

Receber transferência de renda se mostrou o fator que mais influencia negativamente a decisão de trabalhar, visto que essa renda proveniente de outra fonte reduziria a necessidade do salário. Tal fato mostra a importância dos programas de transferência de renda para mudar a realidade dos jovens de baixa renda. Sem a necessidade de trabalhar, os mesmo podem se dedicar à sua formação e assim, no futuro, conseguir melhores colocações no mercado de trabalho. A escolaridade dos pais e a renda familiar também foram fatores de influência negativa.

Quanto à determinação dos rendimentos, os fatores que influenciam positivamente são a idade, escolaridade, ser branco e do sexo masculino, o que mostra que mulheres e não brancos tendem a ocupar cargos com salários mais baixos. Vale ressaltar a importância da escolaridade na determinação dos rendimentos, visto que cada ano a mais de formação escolar aumenta o rendimento médio em 4,45pp. Isso corrobora a importância da educação na redução da pobreza, bem como comprova a teoria do capital humano.

Trabalhar no setor primário e terciário também se mostrou expressivo na determinação dos rendimentos, visto que os salários nesses setores tendem a ser menores na RMR.

Assim sendo, como os jovens participarem do mercado de trabalho é uma realidade, cabe ao poder público dar condições para que esses, mesmo trabalhando, continuem os estudos para garantir melhores oportunidades futuras. Esse é um caminho para melhoria da qualidade de vida da população e crescimento econômico da região.

Por fim, investir na educação e nas condições de acesso e permanência dos jovens na mesma é um passo indispensável rumo à redução das disparidades sociais da RMR. 


\section{Carolina Rodrigues Corrêa}

Universidade Federal de Juiz de Fora, Faculdade de Economia. Governador Valadares/MG, Brasil. carolina.rodcor@gmail.com

\section{João Eustáquio de Lima}

Universidade Federal de Viçosa, Programa de Pós-Graduação em Economia Aplicada. Viçosa/MG, Brasil. jelima@ufv.br

\section{Notas}

(1) A pesquisa da PNAD 2012 foi realizada entre os dias 23 e 29 de setembro de 2012 (Notas técnicas, IBGE).

(2) Para mais informações sobre o procedimento de Heckman, ver Hoffmann e Kassouf (2005); Greene (2003) ou Heckman (1979).

\section{Referências}

ALTONJI, J. G.; BLOM, E. e MEGHIR, C. (2012). Heterogeneity in human capital investments: High school curriculum, college major, and careers. NBER Working Paper, n. 17985.

BARROS, R. P.; HENRIQUES, R. e MENDONÇA, R. (2002). Pelo fim das décadas perdidas: educação e desenvolvimento sustentado no Brasil. Texto para discussão n. 857, Ipea.

BASU, K. e VAN, P. H. (1998). The economics of child labor. American Economic Review, v. 88, n. 3, pp. 412-427.

BECKER, G. S. (1971). The economics of discrimination: an economic view of racial discrimination. Chicago, The University of Chicago Press.

BITOUN, J. et al. (2012). Região Metropolitana do Recife no contexto de Pernambuco no Censo 2010. Observatório das Metrópoles. Recife.

COELHO, A. M. e CORSEUIL, C. H. (2002). Diferenciais salariais no Brasil: um breve panorama. Texto para discussão n. 898. Ipea. Disponível em: <http://www.ipea.gov.br/sites/000/2/livros/ estruturasalarial/capitulo3_diferenciais.pdf>. Acesso em: 11 maio 2015.

FARBER, H. S. e GIBBONS, R. (1996). Learning and wage dynamics. The Quarterly Journal of Economics, v. 111 , n. 4, pp. 1007-1047.

GREENE, W. (2003). Econometric Analysis. Nova Jersey, Prentice Hall. 
HECKMAN, J. J. (1979). Sample selection bias as a specification error. Econometrica. Menasha, v. 47, n. 1, pp. 153-161.

HOFFMANN, R. (2001). "Income distribution in Brazil and the regional and sectorial contrasts". In: GUILHOTO, J. J. M. e HEWINGS, G. J. D. (orgs.). Structure and structural change in the Brazilian economy. Ashgate.

HOFFMANN, R. e KASSOUF, A. L. (2005). Deriving conditional and unconditional marginal effects in log earnings equations estimated by Heckman's procedure. Applied Economics. Londres, v. 37, n. 11, pp. 1303-1311.

IBGE (2012). Pesquisa Nacional por Amostra de Domicílios - PNAD. Disponível em: http://www.ibge. gov.br/home/estatistica/populacao/trabalhoerendimento/pnad2012/microdados.shtm. Acesso em: 9 maio 2014.

LANGONI, C. (1973). Distribuição da renda e desenvolvimento econômico do Brasil. Rio de Janeiro, Expressão e Cultura.

LEONE, E. T. e BALTAR, P. (2013). Mercado de trabalho metropolitano: gênero e diferenças de rendimento, 1992-2002. Revista Gênero, v. 7, n. 1.

LIMA, R. (1980). Mercado de trabalho: o capital humano e a teoria da segmentação. Pesquisa e Planejamento Econômico, v. 10, n. 1, pp. 217-272.

MEDEIROS, B. L. (2011). Diferencial de salários e produtividade no setor de serviços brasileiro. Dissertação de Mestrado. São Paulo, Fundação Getúlio Vargas.

MESQUITA, M. R. (2011). A inserção dos jovens no mercado de trabalho e o acesso aos direitos trabalhistas no Brasil dos anos 2000. Tese de Doutorado. Campinas, Universidade Estadual de Campinas.

MINCER, J. (1974). Schooling, experience, and earnings. National Bureau of Economic Research. Nova York, Columbia University Press.

OIT (2009). Trabalho decente e Juventude no Brasil. Brasília.

ONU BR. (2014) Entrada dos jovens no mercado de trabalho é fortemente marcada por desigualdades sociais. Disponível em: http://www.onu.org.br/oit-entrada-dos-jovens-no-mercado-de-trabalhoe-fortemente-marcada-por-desigualdades-sociais/. Acesso em: 12 maio 2014.

SARAVÍ, G. A. (2009). Juventud y sentidos de pertencia em América Latina: causas y riesgos de fragmentación social. Revista Cepal. Santiago, n. 98.

SCHLINDWEIN, M. M. e KASSOUF, A. L. (2006). Análise da influência de alguns fatores socioeconômicos e demográficos no consumo domiciliar de carnes no Brasil. Revista de Economia e Sociologia Rural, v. 44, n. 3, pp. 549-572.

SCORZAFAVE, L. G. e MENEZES FILHO, N. A. (2001). Participação feminina no mercado de trabalho brasileiro: evolução e determinantes. Pesquisa e Planejamento Econômico. Rio de Janeiro, v. 31, n. 3, pp. 441-477.

SILVA, I. L. F. e OLIVEIRA, R. B. (2007). “Juventudes, educação e trabalho”. In: JEOLÁS, L. S. et al. (orgs.). Juventude, desigualdades e diversidades. Londrina, Eduel. 
SOUZA, A. P.; PONCZEK, V. e OLIVA, B. (2011). Os determinantes do fluxo escolar entre o ensino fundamental e o ensino médio no Brasil. Texto para Discussão FGV 286. São Paulo, Fundação Getúlio Vargas.

SULIANO, D. C. e SIQUEIRA, M. L. (2012). Retornos da educação no Brasil em âmbito regional considerando um ambiente de menor desigualdade. Economia Aplicada, v. 16, n. 1, pp. 137-165.

VERBEEK, M. (2000). A guide to modern econometrics. Chichester, John Wiley \& Sons.

Texto recebido em 29/out/2014

Texto aprovado em 5/mar/2015 
